\title{
Utility of X-ray and indocyanine green fluorescence imaging in detecting hepatocellular carcinoma dissemination on laparoscopic surgery: A case report
}

\author{
SHU SASAKI ${ }^{1}$, MAKOTO TAKAHASHI ${ }^{1}$, TATSUYA HAYASHI $^{1}$, YASUHIRO MORITA ${ }^{1}$, \\ SHIN NAMIKI ${ }^{2}$ and SHINGO ITAGAKI ${ }^{3}$ \\ Departments of ${ }^{1}$ Surgery, ${ }^{2}$ Gastroenterology and Hepatology and \\ ${ }^{3}$ Pathology, Tokyo Metropolitan Tama Medical Center, Fuchu-shi, Tokyo 183-8524, Japan
}

Received July 3, 2021; Accepted September 2, 2021

DOI: $10.3892 / \mathrm{mco} .2021 .2421$

\begin{abstract}
Indocyanine green (ICG) fluorescence imaging is useful for the intraoperative detection of the peritoneal dissemination of hepatocellular carcinoma (HCC). However, in laparoscopic surgery, disseminations cannot be accurately identified unless the camera lens is positioned close to the lesion. The present study describes a case of $\mathrm{HCC}$ dissemination in which the lesions were accurately identified by combining intraoperative fluoroscopy with ICG fluorescence imaging. A 76-year-old male was diagnosed with HCC dissemination. Computed tomography revealed a $9-\mathrm{mm}$ disseminated nodule near the gallbladder. Although transarterial chemoembolization had also been used to treat this lesion, chemoembolization was technically difficult to perform. Therefore, a coil was placed around the lesion to serve as an intraoperative landmark for later laparoscopic resection. Given the potential difficulty of detecting the lesion during laparoscopic surgery, ICG fluorescence imaging was used to determine the approximate location of the dissemination. The lesion exhibited strong fluorescence, which facilitated its complete resection.
\end{abstract}

\section{Introduction}

Indocyanine green (ICG) fluorescence imaging is used intraoperatively to detect the disseminated recurrences of hepatocellular carcinoma (HCC) (1). However, as the tissue penetration of near-infrared light is limited, detecting tumors in deeper sites using this approach is difficult. At our department, a case of minor peritoneal dissemination of $\mathrm{HCC}$ was

Correspondence to: Dr Makoto Takahashi, Department of Surgery, Tokyo Metropolitan Tama Medical Center, 2-8-29 Musashidai, Fuchu-shi, Tokyo 183-8524, Japan

E-mail: makoto_6_c@yahoo.co.jp

Key words: hepatocellular carcinoma, ICG fluorescence imaging, intraoperative fluoroscopy, laparoscopic surgery, peritoneal dissemination encountered, in which the lesions were able to be accurately identified and located by combining intraoperative fluoroscopy with ICG fluorescence imaging during laparoscopic surgery.

\section{Case report}

A 76-year-old male was diagnosed with HCC caused by alcoholic liver cirrhosis. The patient had received percutaneous ethanol injection therapy for S2 and S5 HCC 13 years previously, radiofrequency ablation for $\mathrm{S} 4 / 5$ HCC 6 years ago and S6 HCC 3 years previously.

At one year prior to presentation, computed tomography revealed tumor dissemination on the surface of S6 and transcatheter arterial chemoembolization was performed. It also revealed a 9-mm disseminated nodule near the gallbladder (Fig. 1). Since intrahepatic lesions were well controlled and there was only one recurrent lesion, surgical treatment was suggested to the patient. However, the patient requested a less invasive treatment and thus, initiation of the treatment with transcatheter arterial chemoembolization was first planned. A disseminated nodule was identified by selective imaging of the ileal artery, which branches off from the superior mesenteric artery (Fig. 2A). Chemoembolization was attempted but impaired blood flow to the ileum was a concern. Since it was technically difficult to treat the lesion with chemoembolization, laparoscopic surgical resection was opted for to remove the lesion. A coil was placed in the artery that supplies the ileum near the disseminated nodule to serve as an intraoperative marker (Fig. 2B).

Given the difficulty of identifying small, disseminated lesions during laparoscopic surgery, ICG fluorescence imaging with intraoperative fluoroscopy was used to facilitate identification. ICG $(0.5 \mathrm{mg} / \mathrm{kg})$ was injected intravenously into the patient $24 \mathrm{~h}$ prior to the operation.

With the coil as a landmark, intraoperative fluoroscopy was used to gauge the disseminated nodule's approximate position (Fig. 2C), which was beneath the adipose tissue (Fig. 3A). The Stryker 1588 AIM camera system was utilized for this. ICG fluorescence imaging confirmed the exact position of the lesion (Fig. 3B), which exhibited strong fluorescence. Therefore, it was completely resected laparoscopically. In addition, two 


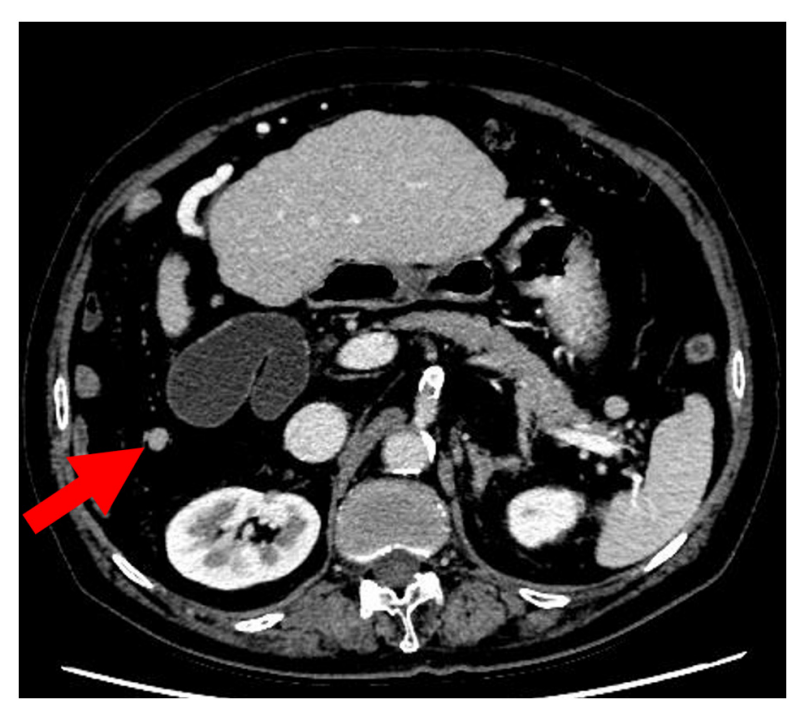

Figure 1. Dissemination of the hepatocellular carcinoma. Computed tomography indicated a 9-mm disseminated nodule around the gallbladder (red arrow).

small lesions on the right diaphragm (Fig. 3C and D) and one small lesion on the liver surface were also detected by fluorography and all were resected. The patient's postoperative course was uneventful and he was discharged on postoperative day 4. Pathologically, all four lesions were diagnosed as HCC disseminations (Fig. 4). At the time of writing this case study, 10 months had passed since the operation and the patient has been alive without any recurrence.

\section{Discussion}

Molecular targeted drug therapy is the standard treatment for advanced HCC with extrahepatic lesions $(2,3)$. However, if intrahepatic lesions are absent or well-controlled, resection of the disseminations may be beneficial. A previous study reported that the cumulative 1-, 3- and 5-year overall survival rates after resection of thoracoabdominal implants were 71 , 44 and $39 \%$, respectively, with a median survival time of 34.5 months (4).

ICG fluorescence imaging has been used in various fields of surgery. In breast cancer surgery, the identification of sentinel lymph nodes using ICG fluorescence imaging has been standardized (5). ICG fluorescence imaging is also used to determine the excision range of non-occlusive mesenteric ischemia (6-8) and evaluate the blood flow at the anastomotic site in colorectal cancer surgery (9). In the field of lung surgery, thoracoscopic lung segment resection using ICG fluorescence imaging has also been performed (10). Nishino et al (11) reported that ICG fluorescence imaging is useful for evaluating arterial blood flow to the stomach in distal pancreatectomy with celiac axis resection. In HCC surgery, ICG fluorescence imaging provides anatomical information during laparotomy and laparoscopic surgery $(12,13)$. Previous studies have reported that ICG fluorescence imaging is a convenient method for intraoperatively detecting extrahepatic HCC metastases $(14,15)$ The uptake of ICG by HCC cells in extrahepatic metastases is similar to that observed in hepatocytes and intrahepatic HCC cells (16). According to Satou et al (1), of the 33 lesions (lung, adrenal gland, lymph node and peritoneum)

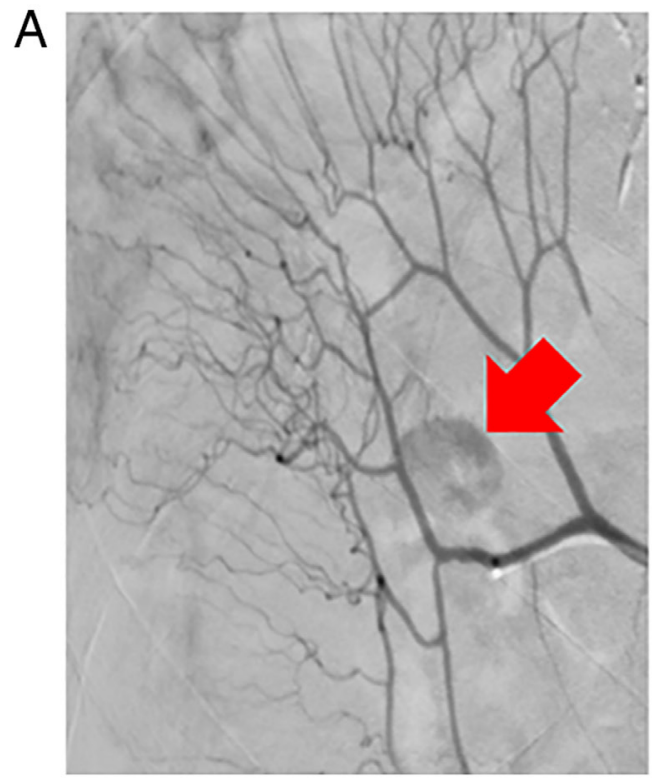

B
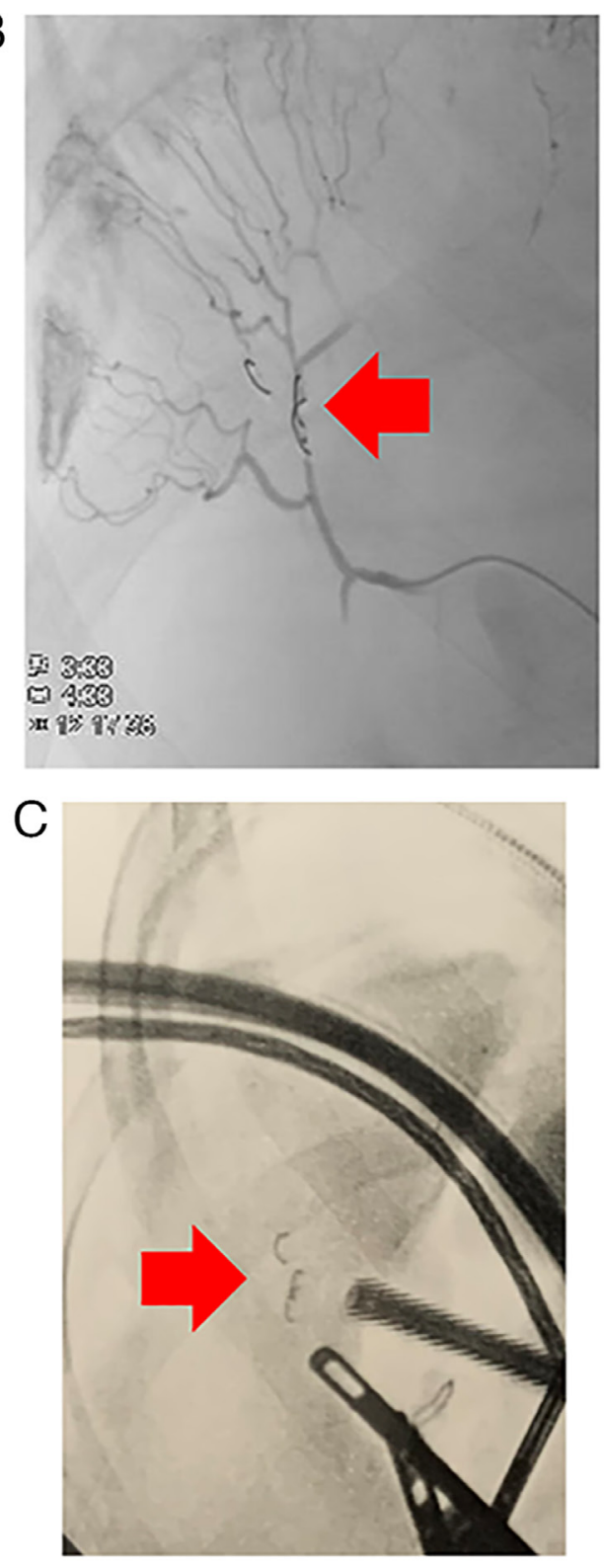

Figure 2. (A) The disseminated nodule was identified by selective imaging from the ileal artery. (B) A coil is placed in the artery near the lesion. (C) Intraoperative fluoroscopy. The lesion is indicated by a red arrow. 
A

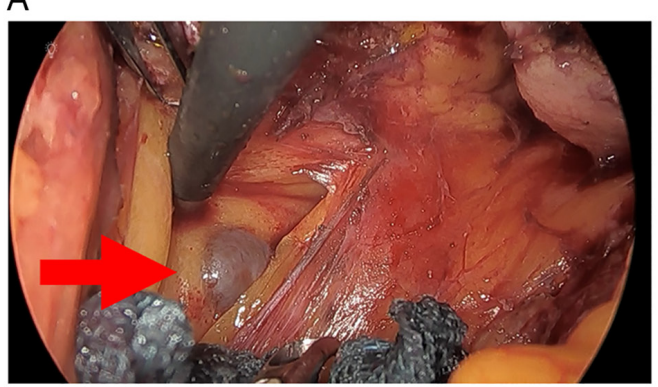

C

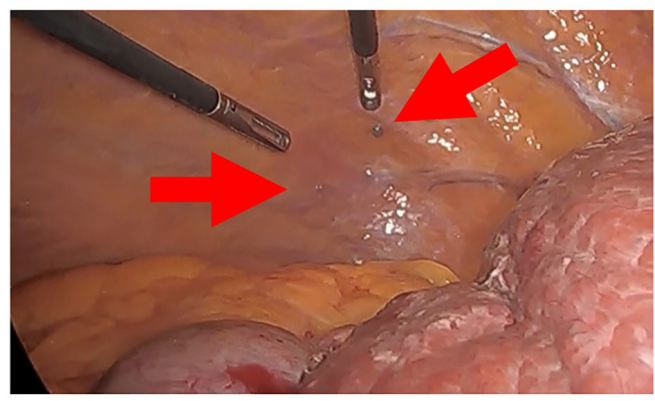

B

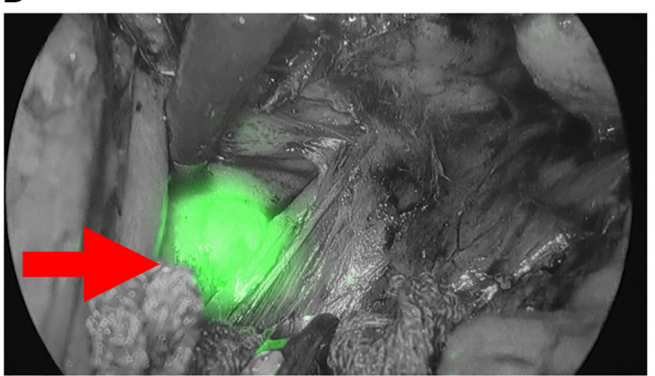

D

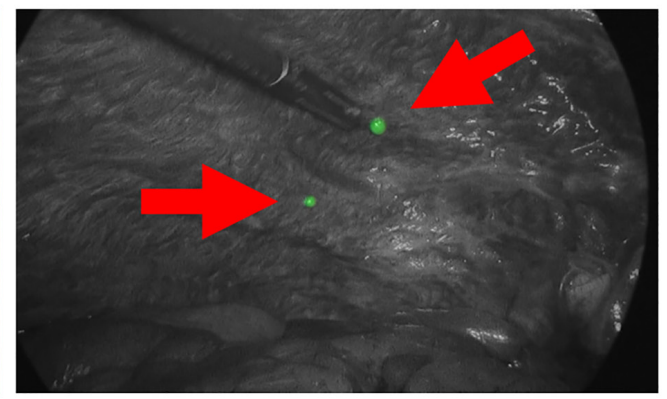

Figure 3. Intraoperative images. (A) Tumor located beneath the adipose tissue. (B) The lesion from A exhibited strong fluorography in ICG mode. (C) Two small lesions, which were not able to be identified preoperatively, were detected on the right diaphragm. (D) The lesions from C exhibited strong fluorography in ICG mode. The lesion is indicated by red arrows.

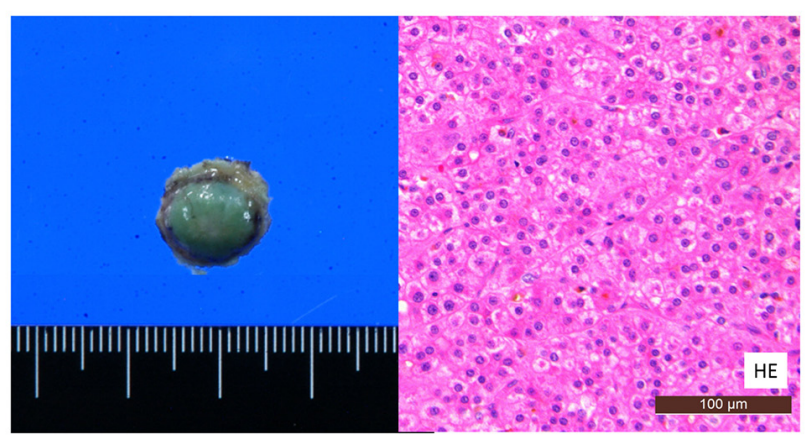

Figure 4. Pathological examination indicated that the resected lesions were disseminations of hepatocellular carcinoma. Left: Image of resected lesion $(\sim 1 \mathrm{~cm})$; right: Histology image with HE staining (scale bar, $100 \mu \mathrm{m})$.

suspected to be extrahepatic metastases of HCC, 26 exhibited fluorescence on ICG and all were metastases of HCC. Of the seven lesions that did not exhibit any fluorescence, one was a metastasis of HCC and six were benign (1).

Ishizawa and Saiura (17) proposed that in patients with intrahepatic HCC, ICG at a dose of $0.5 \mathrm{mg} / \mathrm{kg}$ body weight should be administered within 2 weeks prior to surgery. In addition, they advised against the administration of ICG 1 day prior to surgery to decrease the possibility of false-positive nodules. In patients with extrahepatic HCC, the currently recommended interval ranges from 1 to 5 days (1). However, the duration of ICG retention in extrahepatic HCC may be longer than expected and ICG administration immediately prior to surgery may be permissible due to the lack of uptake by the background tissue (18). In the present case, ICG $0.5 \mathrm{mg} / \mathrm{kg}$ was administered intravenously $24 \mathrm{~h}$ prior to surgery.

Small metastatic nodules are difficult to recognize with the naked eye or on standard laparoscopic view. For extrahepatic
HCC metastases, ICG fluorescence imaging has a positive predictive value of $100 \%$ and a sensitivity of $92 \%$ (1). However, as the tissue penetration of near-infrared light is limited to 5-10 $\mathrm{mm}$ (19), detecting tumors in deeper sites, such as the dorsal site of adipose tissue, using this approach is difficult. To identify deep-seated lesions, a coil may be placed in the artery near the lesion and intraoperative fluoroscopy with ICG fluorescence may be used to facilitate detection. In the present case, the disseminated lesion was able to be identified laparoscopically using this method. Placing a marker, such as a coil, around a tumor may be considered if the tumor is difficult to detect with ICG fluorescence imaging alone. Furthermore, disseminated lesions that cannot be identified preoperatively may be identified using ICG fluorescence imaging.

ICG contains iodine, and there are reports of occasional anaphylactic shock due to administration $(20,21)$. Therefore, it is necessary to be aware of the patient's history of allergies.

In conclusion, the combination of intraoperative ICG fluorescence imaging and fluoroscopy is useful for identifying small, disseminated HCC lesions laparoscopically.

\section{Acknowledgements}

Not applicable.

\section{Funding}

No funding was received.

\section{Availability of data and materials}

The datasets used and/or analyzed during the current study are available from the corresponding author upon reasonable request. 


\section{Authors' contributions}

SS drafted the manuscript. MT revised the manuscript. YM and SN contributed to preoperative checks and diagnoses. SS, MT and TH performed the surgery. YM and SN followed up the patient. SI diagnosed the disease as a pathologist. MT and SS checked and confirmed the authenticity of the raw data. All authors read and approved the final manuscript.

\section{Ethics approval and consent to participate}

Not applicable.

\section{Patient consent for publication}

Written informed consent was obtained from the patient for publication of this case report.

\section{Competing interests}

The authors declare that they have no competing interests.

\section{References}

1. Satou S, Ishizawa T, Masuda K, Kaneko J, Aoki T, Sakamoto Y, Hasegawa K, Sugawara Y and Kokudo N: Indocyanine green fluorescent imaging for detecting extrahepatic metastasis of hepatocellular carcinoma. J Gastroenterol 48: 1136-1143, 2013.

2. Llovet JM, Ricci S, Mazzaferro V, Hilgard P, Gane E, Blanc JF, de Oliveira AC, Santoro A, Raoul JL, Forner A, et al: Sorafenib in advanced hepatocellular carcinoma. N Engl J Med 359: 378-390, 2008.

3. Cheng AL, Kang YK, Chen Z, Tsao CJ, Qin S, Kim JS, Luo R, Feng J, Ye S, Yang TS, et al: Efficacy and safety of sorafenib in patients in the Asia-Pacific region with advanced hepatocellular carcinoma: A phase III randomised, double-blind, placebo-controlled trial. Lancet Oncol 10: 25-34, 2009.

4. Takemura N, Hasegawa K, Aoki T, Sakamoto Y, Sugawara Y, Makuuchi M and Kokudo N: Surgical resection of peritoneal or thoracoabdominal wall implants from hepatocellular carcinoma. Br J Surg 101: 1017-1022, 2014.

5. Sugie T, Kinoshita T, Masuda N, Sawada T, Yamauchi A, Kuroi K, Taguchi T, Bando H, Yamashiro H, Lee T, et al: Evaluation of the clinical utility of the ICG fluorescence method compared with the radioisotope method for sentinel lymph node biopsy in breast cancer. Ann Surg Oncol 23: 44-50, 2016.

6. Nakagawa Y, Kobayashi K, Kuwabara S, Shibuya H and Nishimaki T: Use of indocyanine green fluorescence imaging to determine the area of bowel resection in non-occlusive mesenteric ischemia: A case report. Int J Surg Case Rep 51: 352-357, 2018.

7. Irie T, Matsutani T, Hagiwara N, Nomura T, Fujita I, Kanazawa Y, Kakinuma D and Uchida E: Successful treatment of non-occlusive mesenteric ischemia with indocyanine green fluorescence and open-abdomen management. Clin J Gastroenterol 10: 514-518, 2017.
8. Nitori N, Deguchi T, Kubota K, Yoshida M, Kato A, Kojima M, Kadomura T, Okada A, Okamura J,Kobayashi M, et al: Successful treatment of non-occlusive mesenteric ischemia (NOMI) using the HyperEye Medical System ${ }^{\mathrm{TM}}$ for intraoperative visualization of the mesenteric and bowel circulation: Report of a case. Surg Today 44: 359-362, 2014.

9. Arezzo A, Bonino MA, Ris F, Boni L, Cassinotti E, Foo DC, Shum NF, Brolese A, Ciarleglio F, Keller DS, et al: Intraoperative use of fluorescence with indocyanine green reduces anastomotic leak rates in rectal cancer surgery: An individual participant data analysis. Surg Endosc 34: 4281-4290, 2020.

10. Mun M, Okumura S, Nakao M, Matsuura Y and Nakagawa K: Indocyanine green fluorescence-navigated thoracoscopic anatomical segmentectomy. J Vis Surg 3: 80, 2017.

11. Nishino H, Takano S, Yoshitomi H, Furukawa K, Takayashiki T, Kuboki S, Suzuki D, Sakai N, Kagawa S, Nojima H, et al: Ischemic gastropathy after distal pancreatectomy with en bloc celiac axis resection versus distal pancreatectomy for pancreatic body/tail cancer. Surg Open Sci 1: 14-19, 2019.

12. Ishizawa T, Fukushima N, Shibahara J, Masuda K, Tamura S, Aoki T, Hasegawa K, Beck Y, Fukayama M and Kokudo N: Real-time identification of liver cancers by using indocyanine green fluorescent imaging. Cancer 115: 2491-2504, 2009.

13. Felli E, Ishizawa T, Cherkaoui Z, Diana M, Tripon S, Baumert TF, Schuster C and Pessaux P: Laparoscopic anatomical liver resection for malignancies using positive or negative staining technique with intraoperative indocyanine green-fluorescence imaging. HPB (Oxford): Jun 7, 2021 (Epub ahead of print).

14. Nanashima A, Tominaga T, Sumida $Y$, Tobinaga $S$ and Nagayasu T: Indocyanine green identification for tumor infiltration or metastasis originating from hepatocellular carcinoma. Int J Surg Case Rep 46: 56-61, 2018.

15. He P, Huang T, Fang C, Su S, Tian J, Xia X and Li B: Identification of extrahepatic metastasis of hepatocellular carcinoma using indocyanine green fluorescence imaging. Photodiagn Photodyn Ther 25: 417-420, 2019.

16. Ishizawa T, Masuda K, Urano Y, Kawaguchi Y, Satou S, Kaneko J, Hasegawa K, Shibahara J, Fukayama M, Tsuji S, et al: Mechanistic background and clinical applications of indocyanine green fluorescence imaging of hepatocellular carcinoma. Ann Surg Oncol 21: 440-448, 2014.

17. Ishizawa $\mathrm{T}$ and Saiura A: Fluorescence imaging for minimally invasive cancer surgery. Surg Oncol Clin N Am 28: 45-60, 2019.

18. Yamamura K, Beppu T, Sato N, Kinoshita K, Oda E, Yuki H, Motohara T, Miyamoto H, Komohara Y and Akahoshi S: Complete removal of adrenal metastasis in hepatocellular carcinoma using indocyanine green fluorescent imaging. Anticancer Res 40: 5823-5828, 2020.

19. Ishizawa T, Bandai Y and Kokudo N: Fluorescent cholangiography using indocyanine green for laparoscopic cholecystectomy: An initial experience. Arch Surg 144: 381-382, 2009.

20. Kim M, Lee S, Park JC, Jang DM, Ha SI, Kim JU, Ahn JS and Park W: Anaphylactic shock after indocyanine green video angiography during cerebrovascular surgery. World Neurosurg 133: 74-79, 2020.

21. Chu W, Chennamsetty A, Toroussian R and Lau C: Anaphylactic shock after intravenous administration of indocyanine green during robotic partial nephrectomy. Urol Case Rep 12: 37-38, 2017.

This work is licensed under a Creative Commons Attribution-NonCommercial-NoDerivatives 4.0 International (CC BY-NC-ND 4.0) License. 